\section{(6) OPEN ACCESS}

\title{
UK vaccination schedule: persistence of immunity to hepatitis $B$ in children vaccinated after perinatal exposure
}

\author{
Tom A Yates, ${ }^{1}$ Karthikeyan Paranthaman, ${ }^{1}$ Ly-Mee Yu, ${ }^{2}$ Elizabeth Davis, ${ }^{1}$ Sarah Lang, ${ }^{3}$ \\ Scott J Hackett, ${ }^{4}$ Steven B Welch, ${ }^{4}$ Andrew J Pollard, ${ }^{1,5}$ Matthew D Snape ${ }^{1,5}$
}

\begin{abstract}
${ }^{1}$ Oxford Vaccine Group, Department of Paediatrics, University of Oxford, Oxford, UK

${ }^{2}$ Centre for Statistics in Medicine, University of Oxford, Oxford, UK

${ }^{3}$ Thames Valley Health Protection Unit, Centre for Radiation, Chemical and Environmental Hazards Chilton, UK

${ }^{4}$ Department of Paediatrics, Birmingham Heartlands Hospital, Birmingham, UK ${ }^{5}$ NIHR Oxford Biomedical Research Centre, Oxford, UK
\end{abstract}

\section{Correspondence to} Dr Tom Yates, Oxford Vaccine Group, Centre for Clinical Vaccinology and Tropical Medicine (CCVTM), Churchill Hospital, Old Road, Headington, Oxford OX3 7LE, UK; t.yates@ucl.ac.uk

Received 11 April 2012 Revised 24 January 2013 Accepted 28 January 2013 Published Online First 9 March 2013

\begin{abstract}
Objective To assess persistence of immunity to hepatitis $B(\mathrm{HBV})$ in primary school children vaccinated following perinatal exposure.

Design Serological survey.

Setting Five UK sites (Berkshire East, Birmingham, Buckinghamshire, Milton Keynes and Oxfordshire).

Participants Children from 3 years 4 months to 10 years of age (mean age 6.2 years), vaccinated against HBV from birth following perinatal exposure.
\end{abstract}

Interventions $A$ single booster dose of the paediatric formulation of a recombinant HBV vaccine.

Main outcome measures Titres of antibody against hepatitis B Surface Antigen (anti-HBs) measured immediately before and 21-35 days after the HBV vaccine booster.

Results Prebooster anti-HBs titres were $>10 \mathrm{mlU} / \mathrm{ml}$ in $84.6 \%$ of children ( $n=26 ; 95 \% \mathrm{Cl} 65.1$ to $95.6 \%$ ). All children $(n=25,95 \% \mathrm{Cl} 86.3$ to $100 \%)$ had titres $>100 \mathrm{mIU} / \mathrm{ml}$ after the booster.

Conclusions This study of antibody persistence among UK children born to hepatitis B infected women, immunised with a 3-dose infant schedule with a toddler booster suggests sustained immunity through early childhood. These data should prompt further studies to address the need for a preschool booster. Trial registration Eudract Number 2008-004785-98.

Children born to women with detectable hepatitis B surface antigen (HBsAg) are at very high risk of becoming chronic hepatitis B virus (HBV) carriers. ${ }^{1}$ An accelerated course of $\mathrm{HBV}$ vaccine initiated at birth is effective at reducing transmission, ${ }^{2}$ and is universally recommended for infants of $\mathrm{HBsAg}$ positive mothers. ${ }^{13}$

Due to low disease prevalence, immunisation against HBV in the UK is currently targeted at specific 'at-risk' groups rather than being universal. Children born to HBV infected mothers are immunised at 0, 1, 2 and 12 months of age. A 2006 update to the UK immunisation guidelines ${ }^{3}$ explicitly recommended a 'preschool' booster dose of HBV vaccine be added to this schedule. Internationally, there is consensus that there are insufficient data to recommend booster doses in immunocompetent children vaccinated following perinatal exposure. ${ }^{14}$ To the best of our knowledge, no other countries recommend HBV vaccine boosters in perinatally exposed children.

To investigate the need for this 'preschool' booster we studied anti-HBsAg (anti-HBs) IgG

\section{What is already known on this topic}

- Children born to women with detectable hepatitis B surface antigen ( $\mathrm{HBsAg}$ ) are at very high risk of becoming chronically infected with hepatitis $B$ virus (HBV).

- An accelerated course of HBV vaccination from birth is effective at reducing transmission and universally recommended for infants of $\mathrm{HBsAg}$ positive mothers.

- Current UK guidelines recommend that children, with ongoing exposure to HBV infection, receive a preschool HBV vaccine booster.

\section{What this study adds}

- This cohort of perinatally exposed UK children, immunised at 0, 1, 2 and 12 months of age, retained immunity against HBV through early childhood.

- There is a need for further research into the necessity of the currently recommended preschool booster dose of HBV vaccine for children immunised with an accelerated course of HBV vaccine in infancy.

titres in children immunised with the current $(0,1$, 2, 12 month) UK infant schedule before and after a booster dose of HBV vaccine in mid-childhood.

\section{POPULATION, MATERIALS AND METHODS \\ Study population}

Letters were sent by clinicians with duty of care to parents of children who had commenced a course of HBV vaccination at birth. Children were identified from local records at three primary care trusts in the Thames Valley, and at the departments of paediatrics at the John Radcliffe Hospital, Oxford, and Heartlands Hospital, Birmingham. The letters informed parents of the recommendation for a preschool HBV vaccine booster, offering them the option of it being administered through this clinical trial.

To be eligible for the study, children had to be aged between 3 years 4 months and 10 years (inclusive), to have received a dose of $\mathrm{HBV}$ vaccine in the first week of life, but not within the preceding 
2 years, and to remain potentially exposed to HBV within the household. Children with significant renal or hepatic impairment, life-limiting illness or evidence of HBV infection on a previous blood test were excluded. Children were enrolled between April 2009 and January 2011.

\section{Study design}

Approvals were obtained from Oxfordshire Research Ethics Committee C (OxREC08/H0606/136), the Medicines and Healthcare Regulatory Agency and relevant National Health Service (NHS) bodies.

Informed signed consent was obtained from parents of all participants.

Children in the study received a single $0.5 \mathrm{ml}$ dose of the paediatric formulation of Engerix B (GlaxoSmithKline, Brentford, UK), a recombinant vaccine $(10 \mu \mathrm{g}$ of $\mathrm{HBsAg}$ adsorbed onto aluminium hydroxide). This was given as an intramuscular injection into the deltoid area via a $25 \mathrm{~mm} 23$ gauge needle.

Blood samples (up to $6 \mathrm{ml}$ ) were obtained immediately before and then 21-35 days following vaccination. Following study completion, information on participants' receipt of hepatitis B immunoglobulin (HBIG) and maternal HBeAg status was obtained, where available, for a posthoc analysis.

\section{Laboratory methods}

Blood samples were stored at $2-8^{\circ} \mathrm{C}$ within $2 \mathrm{~h}$ of sampling. Within $24 \mathrm{~h}$ of sampling, they were centrifuged at $3000 \mathrm{~g}$ for $10 \mathrm{~min}$ and sera stored at $\leq-18^{\circ} \mathrm{C}$. Sera were tested by ELISA at the microbiology laboratory, John Radcliffe Hospital, Oxford, for anti-HBs IgG, IgG against HBV core antigen (anti-HBc) and, as needed, HBsAg concentrations.

\section{Statistical methods}

Two analyses were undertaken: an 'intention-to-treat' (ITT) analysis including all children, and a 'per-protocol' (PP) analysis including only children who had received four doses of vaccine with no significant delays to their schedule (ie, three doses by 6 months and four doses by 18 months of age).

Using STATA, V.11 (StataCorp, College Station, USA), 95\% binomial proportion CIs were calculated. We considered two thresholds-anti-HBs $>10 \mathrm{mIU} / \mathrm{ml}$ prevaccine booster, a measure of antibody persistence, and anti-HBs $>100 \mathrm{mIU} / \mathrm{ml}$ postvaccine booster, a measure of immune memory. Calculation of geometric mean concentrations of anti-HBs IgG was not performed, as exact concentrations were not determined where titres were $>1000 \mathrm{mIU} / \mathrm{ml}$.

\section{RESULTS}

\section{Participant characteristics}

Twenty-eight children with mean age 6.2 years (range 3.410.5 years) were enrolled. Nineteen $(68 \%)$ were over the age of 5 years at enrolment, and were therefore overdue for their HBV 'preschool' booster. Fourteen (50\%) were male. There were 15 Asian, 5 black children and 2 Caucasian children, plus 6 children of other or mixed ethnicity. The cohort included one set of three siblings and eight sets of two siblings. Twenty-four children $(86 \%)$ had received a four-dose infant schedule with no significant delays, so were included in the PP analysis. The ITT analysis included all 28 children (figure 1).

Of the 23 children on whom data were available on receipt of HBIG, 4 (17\%) had received this. Of the 22 children for whom there were available data on maternal HBeAg status, 2 (9\%) were positive. Three of the five children for whom there were no data regarding receipt of HBIG were born to $\mathrm{HBeAg}$ negative mothers, so were unlikely to have received it.

\section{Protocol deviations}

Four blood samples were documented to have been separated up to $16 \mathrm{~h}$ later than allowed in the protocol. It was considered unlikely this would have significantly affected the assay. The data were therefore included in the analysis.

\section{High-dose hook effect}

Falls in anti-HBs titres between prebooster and postbooster samples in two participants raised concern that the assay might be influenced by a 'high-dose hook' effect ${ }^{5}$ resulting in spuriously low antibody measurements. Repeat assays following dilution, performed by the John Radcliffe microbiology laboratory and the assay manufacturer (Siemens Healthcare Diagnostics, Deerfield, USA), revealed comparable baseline and elevated postbooster anti-HBs titres $>1000 \mathrm{mIU} / \mathrm{ml}$ in these children. To confirm assay reliability, Siemens repeated 45 of the 51 anti-HBs assays finding no difference in the proportion of assays $>10 \mathrm{mIU} / \mathrm{ml}$ prebooster or $>100 \mathrm{mIU} / \mathrm{ml}$ postbooster. In view of this, the results from John Radcliffe microbiology laboratory are presented, with the two spuriously low measurements corrected to $>1000 \mathrm{mIU} / \mathrm{ml}$.

\section{Baseline anti-HBs concentrations}

ITT analysis found 22 of the 26 children with samples had anti-HBs antibody concentrations $>10 \mathrm{mIU} / \mathrm{ml}$ prior to their preschool booster dose of HBV vaccine $(84.6 \%$; $95 \% \mathrm{CI}$ 65.1 to $95.6 \%)$. For the PP analysis, the proportion was $86.4 \%$ ( $n=22 ; 95 \%$ CI 65.1 to $97.1 \%)$. No child had undetectable anti-HBs concentrations before the booster, the lowest level being $3.24 \mathrm{mIU} / \mathrm{ml}$ (figure 2).

\section{Response to booster}

In an ITT analysis, all 25 children with postbooster samples had anti-HBs concentrations $>100 \mathrm{mIU} / \mathrm{ml} \quad(95 \%$ CI 86.3 to $100 \%$ ) (figure 2). The $95 \%$ CI for the PP analysis was 83.9-100\% $(n=21)$.

\section{Evidence of infection}

One child was positive for anti-HBc at low levels with negative assays for HBsAg and antibody against $\mathrm{HBV}$ e-antigen ( $\mathrm{HBeAg}$ ). Enquiries revealed this child had had equivocal anti-HBc antibody levels when aged 1 year, suggesting transient infection occurred within the first year of life. Subsequent testing for HBV DNA by PCR was negative, suggesting no current hepatitis B infection. Anti-HBs titres from this child $-14.9 \mathrm{mIU} / \mathrm{ml}$ prevaccine and $935.7 \mathrm{mIU} / \mathrm{ml}$ postvaccine booster-were included in the analysis.

\section{DISCUSSION}

Most data on childhood persistence of anti-HBs and the incidence of 'breakthrough' HBV infections following an accelerated course of HBV immunisation are from high-prevalence countries, ${ }^{6}$ where boosting of anti-HBs titres through natural exposure $^{7}$ may be more frequent. With lower rates of natural boosting in low-prevalence countries, such as the UK, anti-HBs concentrations may decline more rapidly. However, children born to HBsAg positive mothers in this setting may remain at ongoing risk of infection from household exposure.

In this small study, children immunised following perinatal exposure, according to current UK recommendations, were all shown to have immunity that had persisted at or beyond the age at which they became eligible for their preschool HBV 
Figure 1 Summary of the recruitment process.

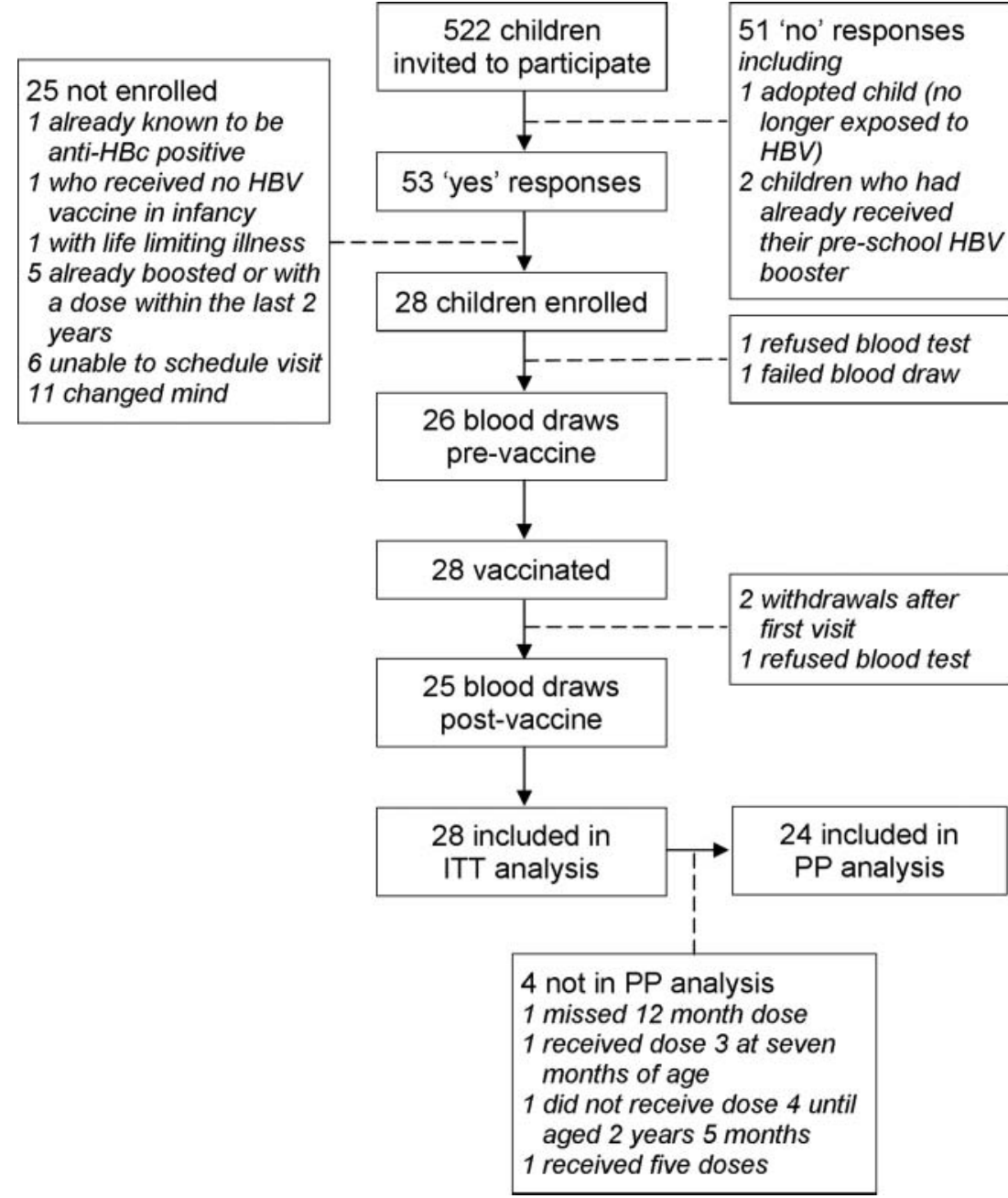

vaccine booster. Most children retained anti-HBs concentrations $>10 \mathrm{mIU} / \mathrm{ml}$ prebooster. After a booster, all mounted strong anti-HBs responses $(>100 \mathrm{mIU} / \mathrm{ml})$.

These findings contrast with those of Boxall et al, ${ }^{8}$ the only other UK study to address this question, who found anti-HBs

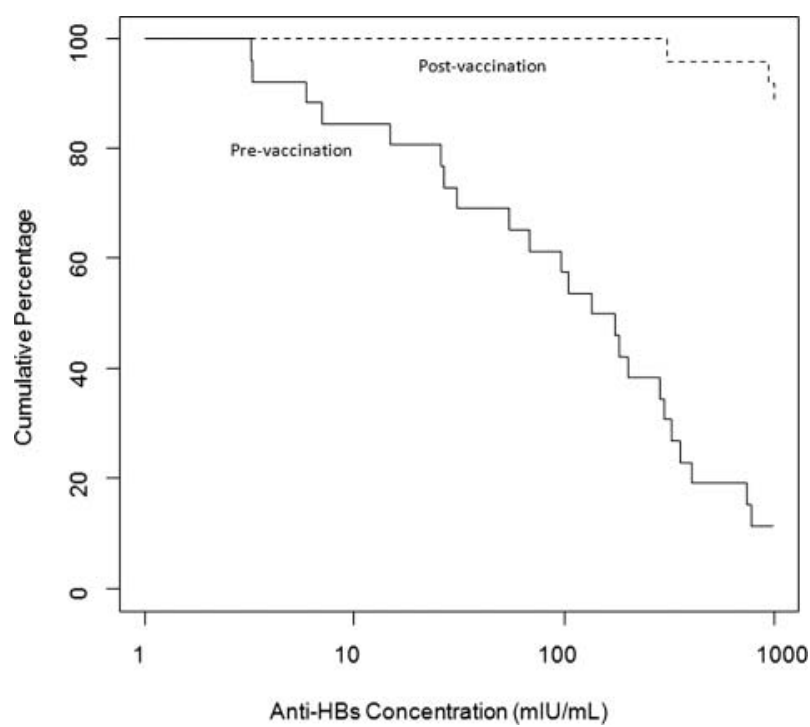

Figure 2 The data included in the intention-to-treat analysis, presented as a reverse cumulative distribution curve.
IgG concentrations $\geq 10 \mathrm{mIU} / \mathrm{ml}$ in only half the adolescents immunised in infancy with an accelerated course $(0,1,2$, 6 months) of HBV vaccine.

The mean age of participants in Boxall's study was 14.5 years, compared with 6.2 years in the current study. A younger cohort (mean age 11.7 years) was also described in the Boxall study with similarly low prebooster anti-HBs titres. The vaccine history of this younger cohort was not described, but these children are likely to have received their last dose at 6 months of age, finishing earlier than children in the current study, who were mostly immunised according to the UK $0,1,2$ and 12-month schedule. It is possible that the difference between our data and those in the Boxall study ${ }^{8}$ simply reflect waning of antibody with age, although, obviously, there are numerous other factors involved.

It is also striking that, in the Boxall study, $16 \%$ of participants had both baseline titres $<10 \mathrm{mIU} / \mathrm{ml}$ and failed to mount a 'memory' response when given a further dose of HBV vaccine (defined as a postvaccination anti-HBs titre $>100 \mathrm{mIU} / \mathrm{ml}$ ). Similar results were seen in the younger cohort in the Boxall study, and these contrast with our findings that all children had IgG $>100 \mathrm{mIU} / \mathrm{ml}$ after the booster dose. In high-prevalence areas, loss of 'memory' response has been seen in some other cohorts of adolescents vaccinated, using various schedules, in infancy. ${ }^{6}$

As well as being younger, children in the current study received their last dose of vaccine at 12 months of age or older (as per current UK guidelines), rather than 6 months of age, as in the Boxall study. It is possible the higher percentage of 
participants with anti-HBs levels $<10 \mathrm{mIU} / \mathrm{ml}$ and the apparent loss of immunological memory in the Boxall study is related to the relative immunological immaturity of these children when they received their last dose.

Finally, a lower proportion of children in the current study received HBIG with their first dose of vaccine than in the study by Boxall et al. ${ }^{8}$ Boxall et al reported that receipt of HBIG might be associated with loss of immunological memory. Although the immunological basis for this observation is still unclear, it is plausible that HBIG given at the same time as the first dose might bind the vaccine antigens and, thus, affect immune priming.

A recent Cochrane review confirmed the lack of high-quality randomised clinical trials (RCTs) to evaluate the need for booster doses of HBV vaccine. ${ }^{9}$ One study ${ }^{10}$ of Senegalese children vaccinated in infancy-some who received a school-age HBV vaccine booster $(n=41)$ and some who did not $(n=51)-$ found that by the age of 9-12 years, the booster significantly reduced the proportion of children with detectable anti-HBc with no impact on HBsAg positivity. This suggests some protection against hepatitis B infection, but the relevance of these data to a low-incidence country is uncertain.

\section{Correlates of protection against HBV infection}

An anti-HBs IgG concentration $<10 \mathrm{mIU} / \mathrm{ml}$ after a primary course of HBV vaccine predicts future vaccine failure. ${ }^{17}{ }^{11}$ To what extent this is due to protection mediated by these antibodies rather than being simply a marker of the strength of vaccine response is not known.

In Gambian children vaccinated in infancy and then followed to age 7 years, waning of anti-HBs concentrations was associated with increased risk of core antibody reversion, a marker of transient infection. ${ }^{11}$ However, multiple studies of children vaccinated in infancy in high-prevalence countries, some with more than 20 years follow-up, have shown that children who initially responded to immunisation remain protected against HBV infection even if anti-HBs concentrations fall below $10 \mathrm{mIU} / \mathrm{ml}^{6}{ }^{6}$ A growing body of evidence suggests this may be true in low-prevalence countries as well. ${ }^{8}{ }^{12-14}$ This protection is thought to be mediated by cell-mediated immunity ${ }^{4}$ and/or anamnestic antibody responses. 4

Citing these observational data, the European Consensus Statement on hepatitis B vaccine booster doses states that there is no evidence to support the use of boosters in immunocompetent children or adolescents. ${ }^{4}$

Many studies have used a dose of $\mathrm{HBV}$ vaccine as a probe to assess immunological memory, defined as a rise in anti-HBs concentrations from $<10 \mathrm{mIU} / \mathrm{ml}$ to either $>10 \mathrm{mIU} / \mathrm{ml}^{4}{ }^{4}{ }^{9}$ or $>100 \mathrm{mIU} / \mathrm{ml}^{8}$ postimmunisation. It is believed that individuals able to mount a memory response to vaccine would mount such a response in the context of natural HBV challenge. Given HBV's long latency period, it is argued that this should be sufficient to clear the infection. ${ }^{4}{ }^{7}$ However, there is no published literature attempting to correlate memory defined in this manner with rates of subsequent breakthrough infection.

Therefore, there is a growing body of evidence ${ }^{6}$ suggesting that protection against HBV infection persists after antibody wanes, and no data correlating loss of a 'memory response' following boosting with future susceptibility to chronic infection. Given the paucity of knowledge about correlates of long-term protection, active surveillance for breakthrough infections may be most informative. We support calls ${ }^{9}$ for RCTs addressing the need for booster doses of HBV vaccine in children born to mothers with hepatitis $B$ infection.

\section{Limitations}

Despite sustained efforts, we were able to recruit a relatively small number of participants to this study, thus limiting our ability to draw wider conclusions from these data. Also, the high number of sibling groups limited the genetic and environmental diversity of the participants, an effect not accounted for in our estimates of protection. It is likely our participants did not fully reflect the population of at-risk children, partly as invitations to participate were not translated into languages other than English. Almost all participants in the current study had received four doses of HBV vaccines by the age of 24 months, whereas the comparative figure for children born between April and June 2010 in England was only 63\%. ${ }^{15}$ It is known that 'at-risk' children eligible for HBV vaccination are liable to be incompletely immunised, and are frequently lost to follow-up. Children with incomplete immunisations may not maintain anti-HBs IgG concentrations to the extent noted in the current study.

\section{Does the 'preschool' booster dose of HBV vaccine have a role in ensuring completion of the infant immunisation schedule?}

Besides concerns about waning of vaccine-induced antibody and immune memory, a key rationale for introducing the preschool booster was that it would 'provide the opportunity to check whether the child was properly followed-up in infancy.' However, it is the years prior to the preschool booster that is the period of greatest risk. A recent review of 85 English children with diagnosed chronic HBV infection (59 of whom were born in England) emphasised that horizontal transmission of HBV infection is a rare event compared with vertical transmission with incomplete primary immunisation. ${ }^{16}$ Administering overdue primary immunisations at the preschool age could, therefore, only impact on the low risk of horizontal transmission, unlike timely uptake of the infant course, ${ }^{15}$ a goal that has been shown to be achievable within the NHS. ${ }^{17} 18$

\section{CONCLUSION}

Although our study is too small to inform policy by itself, our findings suggest that children born to hepatitis B infected women, immunised with a 3-dose infant schedule and toddler booster appear to maintain immunity in early childhood. Further larger studies on the need for a preschool booster, and the persistence of protection into late childhood in completely and incompletely immunised children, are warranted.

Acknowledgements This study could not have been conducted without the participants and their families. We are also grateful to Emma Plested at Oxford Vaccine Group, Pam Griffiths at Buckinghamshire PCT, Erika Lamb at Milton Keynes PCT, Marilyn Lansley at Berkshire East PCT, Rachel Sandford and Annie Roberts at the John Radcliffe Hospital, Oxford, Karen Evans and Jacqueline Daglish at Birmingham Heartlands Hospital and the staff at MIDRU (http://www.midru.com) for their support. AJP is a Jenner Investigator and a James Martin Senior Fellow. MDS receives salary support from the NIHR Oxford Biomedical Research Centre.

Contributors KP, SL, MDS and AJP conceived and designed the study. TAY, KP and ED oversaw the day-to-day running of the study. TAY, KP, ED, SBW, SJH and MDS conducted study visits. The data were analysed by TAY and LMY. TAY wrote the manuscript with all authors commenting on drafts. TAY is guarantor.

Funding This work was supported by the NIHR Oxford Biomedical Research Centre and GlaxoSmithKline Biologicals. The funders had no involvement in the design or conduct of the study, in the data analysis or in the preparation of this manuscript for publication. The data were held by the authors, not the funders.

Competing interests SL has undertaken paid work for vaccine manufacturers for provision of travel health training and attendance at advisory group meetings. AJP and MDS have conducted clinical trials on behalf of Oxford University sponsored by manufacturers of vaccines. AJP and MDS do not accept any personal payments from vaccine manufacturers: grants for support of educational activities are paid to an educational/administrative fund held by the Department of Paediatrics, Oxford 
University. MDS has received support from vaccine manufacturers to attend academic conferences. ED, SBW, SJH, KP, LMY and TAY declare no conflicts of interest besides funding received for this study.

Ethics approval Oxfordshire Research Ethics Committee C.

Provenance and peer review Not commissioned; externally peer reviewed.

Meetings These data were presented as a poster at the annual meeting of the European Society of Paediatric Infectious Disease, The Hague, 7-11 June 2011, and orally at the UK Paediatric Vaccine Group meeting, St George's Medical School, London, on 31 January 2012.

Open Access This is an Open Access article distributed in accordance with the Creative Commons Attribution Non Commercial (CC BY-NC 3.0) license, which permits others to distribute, remix, adapt, build upon this work non-commercially, and license their derivative works on different terms, provided the original work is properly cited and the use is non-commercial. See: http://creativecommons.org/licenses/by-nc/ 3.01

\section{REFERENCES}

1 World Health Organisation. Hepatitis B vaccines. Wkly Epidemiol Rec 2009;84:405-19.

2 Lee C, Gong Y, Brok J, et al. Hepatitis B immunisation for newborn infants of hepatitis B surface antigen-positive mothers. Cochrane Database Syst Rev 2006;(2): CD004790.

3 Department of Health. Immunisation against infectious disease, 2006. http://www. dh.gov.uk/prod_consum_dh/groups/dh_digitalassets/documents/digitalasset/ dh 116622.pdf (accessed 12 Jan 2012).

4 European Consensus Group on Hepatitis B Immunity. Are booster immunisations needed for lifelong hepatitis B immunity? Lancet 2000;355:561-5.

5 Butler J, Chambers SM. Immunoassay in clinical biochemistry. In: Burnett D, Crocker J, eds. The science of laboratory diagnosis. 2nd edn. Chichester: John Wiley \& Sons, 2005: 373-80.

6 Leuridan E, Van Damme P. Hepatitis B and the need for a booster dose. Clin Infect Dis 2011;53:68-75.
7 West DJ, Calandra GB. Vaccine induced immunologic memory for hepatitis B surface antigen: implications for policy on booster vaccination. Vaccine 1996:14:1019-27.

8 Boxall EH, A Sira J, El-Shuhkri N, et al. Long-term persistence of immunity to hepatitis B after vaccination during infancy in a country where endemicity is low. $J$ Infect Dis 2004;190:1264-9.

9 Poorolajal J, Mahmoodi M, Haghdoost A, et al. Booster dose vaccination for preventing hepatitis B. Cochrane Database Syst Rev 2010;(11):CD008256.

10 Coursaget $P$, Leboulleux $D$, Soumare $M$, et al. Twelve-year follow-up study of hepatitis B immunization of Senegalese infants. J Hepatol 1994;21:250-4.

11 Jack $A D$, Hall $A J$, Maine $N$, et al. What level of Hepatitis $B$ antibody is protective? J Infect Dis 1999;179:489-92.

12 Mele $A$, Tancredi $F$, Romanò $L$, et al. Effectiveness of hepatitis $B$ vaccination in babies born to hepatitis B surface antigen-positive mothers in Italy. J Infect Dis 2001;184:905-8

13 Bracciale L, Fabbiani M, Sansoni A, et al. Impact of hepatitis B vaccination in children born to HBsAg-positive mothers: a 20-year retrospective study. Infection 2009:37:340-3.

14 Roznovsky L, Orsagova I, Kloudova A, et al. Long-term protection against hepatitis B after newborn vaccination: 20-year follow-up. Infection 2010:38:395-400.

15 Health Protection Agency. Quarterly vaccination coverage statistics for children aged up to five years in the UK (COVER programme): April to June 2012. Health Protection Report 2012;6:7-13.

16 Flood J, Amirthalingam G, Ramsay ME, et al. The diagnosis of chronic Hepatitis B infection among children born in England after introduction of universal antenatal HBV screening programme. Poster presented at the European Society of Paediatric Infectious Disease Meeting, The Hague, June 2011. http://www.kenes.com/ espid2011/cd/pdf/P774.pdf (accessed 20 Jul 2012).

17 Larcher VF, Bourne J, Aitken C, et al. Overcoming barriers to hepatitis B immunisation by a dedicated hepatitis B immunisation service. Arch Dis Child 2001:84:114-19.

18 Delamoth T. BMJ Group awards. Secondary care team of the year: the magic of teamwork. BMJ 2011:342:d2320. 